$15^{\text {th }}$ International Conference on

AEROSPACE SCIENCES \& AVIATION TECHNOLOGY,

$\boldsymbol{A S A T}$ - 15 - May 28 - 30, 2013, Email: asat@mtc.edu.eg,

Military Technical College, Kobry Elkobbah, Cairo, Egypt,

Tel: +(202) 24025292-24036138, Fax: +(202) 22621908

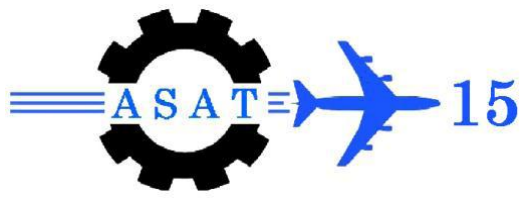

\title{
Flight Testing for SUAV Using Log Analysis of Low-Cost Autopilot
}

\author{
M. K. Zakaria ${ }^{*}$, A. M. Hammad, M. A. Moattasem $\}^{\dagger}$
}

\begin{abstract}
In this paper, we discuss the process of integrating an Autopilot with Unmanned Aerial Vehicle sensors and actuators to perform full Autonomous mission. Unmanned aerial vehicles (UAVs) play an important role in the current military operations in the recent years with its great advantages for autonomous flying capability that provides much less manpower, cost and less risk to human lives. Also there are many potential applications for UAVs in civilian environment like photography, agriculture planning and many others. To gain an autonomous flying requires many flight testing and evaluations to decide to use certain airframe aircraft integrated with the autopilot in a required application. Using a low cost Autopilot, a MATLAB program was written in order to analyze log files for every flight test in different SUAV modes (Manual, Stabilize and Auto). Tuning the autopilot PID control gains during flight testing has a significant effect on aircraft flight stability that requires less iteration until it reaches an adequate value which requires an intensive work.
\end{abstract}

\section{Introduction}

Design and development of UAV system is an awkward and difficult challenge. This activity requires pre-flight and post-flight checks to enhance the UAV system performance. As any Aircraft maintenance group working on any civilian or military aircraft, pre-flight checks must be done before taking a decision to make a required mission. A Post flight evaluation for the UAV performance during the flight must be done; therefore a huge quantity of data must be monitored and analyzed as servo-controlled loops, speed, altitude, battery power consumption, GPS fix (Sat. counts). This activity requires precise tools and evaluation methods to analyze the UAV parameters. In this paper we develop a set of data evaluations procedures using MATLAB and C\# to give a good insight of available information and then assess the performance of the flight.

\section{2- UAS Case Study}

\subsection{Sakr 2 UAV Platform}

The design and construction of the SUAV was completed in early march 2011[1]. As demonstrated in Fig. 1. The SUAV consisted of a Tailless design, powered by a single electric engine, with Pusher arrangement.

\footnotetext{
M_y_zakaria@hotmail.com

$\dagger \quad$ Egyptian Armed Forces, Egypt.
} 


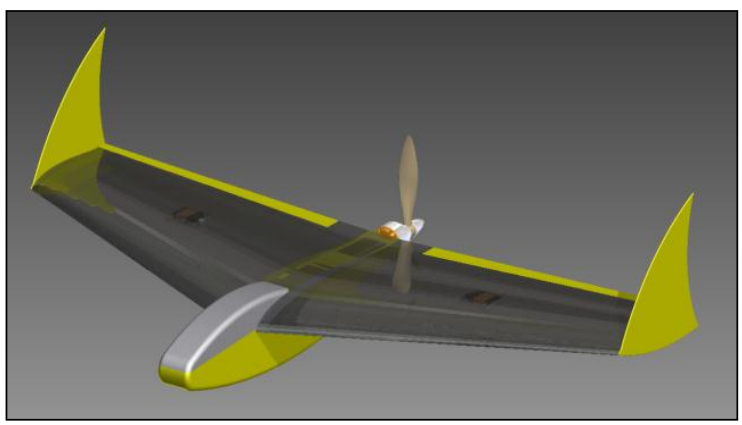

\section{SAKR 2 Specification}

Span: $120 \mathrm{~cm}$

Length: $75 \mathrm{~cm}$

Height: $32 \mathrm{~cm}$

MTOW: $2.6 \mathrm{~kg}$

Range: $10 \mathrm{~km}$

Endurance: $25 \mathrm{~min}$

Fig. 1 UAS case study platform specifications (SAKR 2 SUAV)

\section{Low-Cost Autopilot}

A complete flight test system consists of several electronics and sensors components. For our purposes, the flight test system consisted of the microcontroller, the navigation subsystem and the telemetry subsystem, Fig. 2.

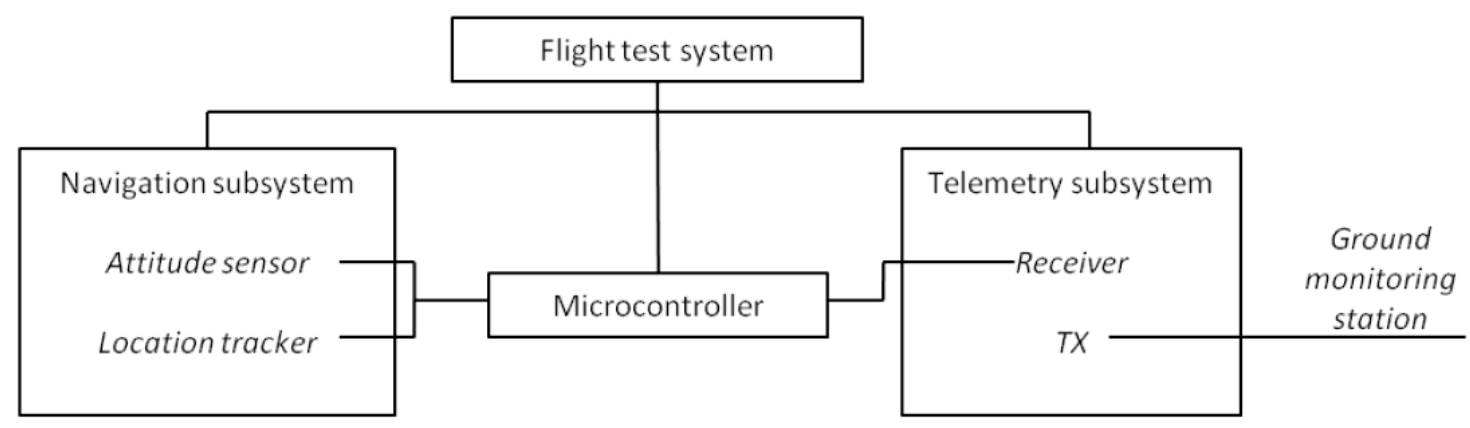

Fig. 2 Flight test system

The microcontroller is the central component, which reads in inputs from pilot's control, navigation and telemetry. It also holds the autopilot code that executes the control laws and computes the commands. The navigation system will require appropriate instruments that can tell its position and measure its attitude. By tracking the aircraft's position and maintaining a stable attitude (orientation in roll, pitch and yaw angles), the aircraft can navigate safely from point to point. While tracking these parameters, a telemetry system must be incorporated so that the data can be recorded for further analysis. Together with a ground monitoring station (Laptop), a telemetry system allows remote monitoring or measurement.

Ardupilot-Mega, [2], is an open source autopilot platform created by DIY Drones. The hardware consists of the core autopilot board and various sensors and accessories that can be added for extra functionality. The board is displayed in Fig. 3. To be able to use it the way it is intended to be used, we had to do some soldering of pins and a few cables, all of which is thoroughly described in [1]. Both hardware and the software are open source. 


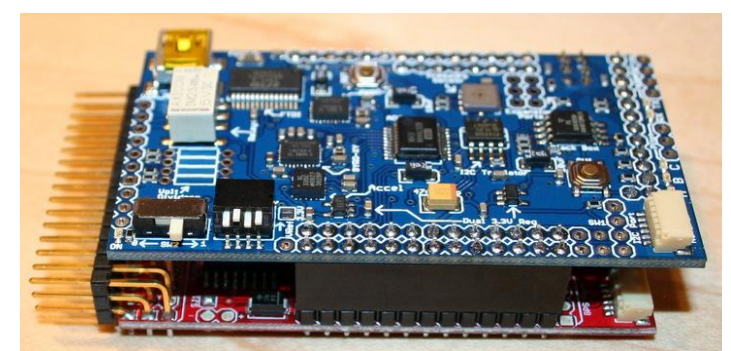

Fig. 3 The ArduPilot-Mega board

\subsection{Fixing Autopilot and Sensors Onboard UAV}

In order to get a better understanding of the implemented algorithm, we proceed by describing the control structure implemented in the routine, Fig. 4. The control structure considered for stabilizing and navigating the plane is a cascaded structure, [3].

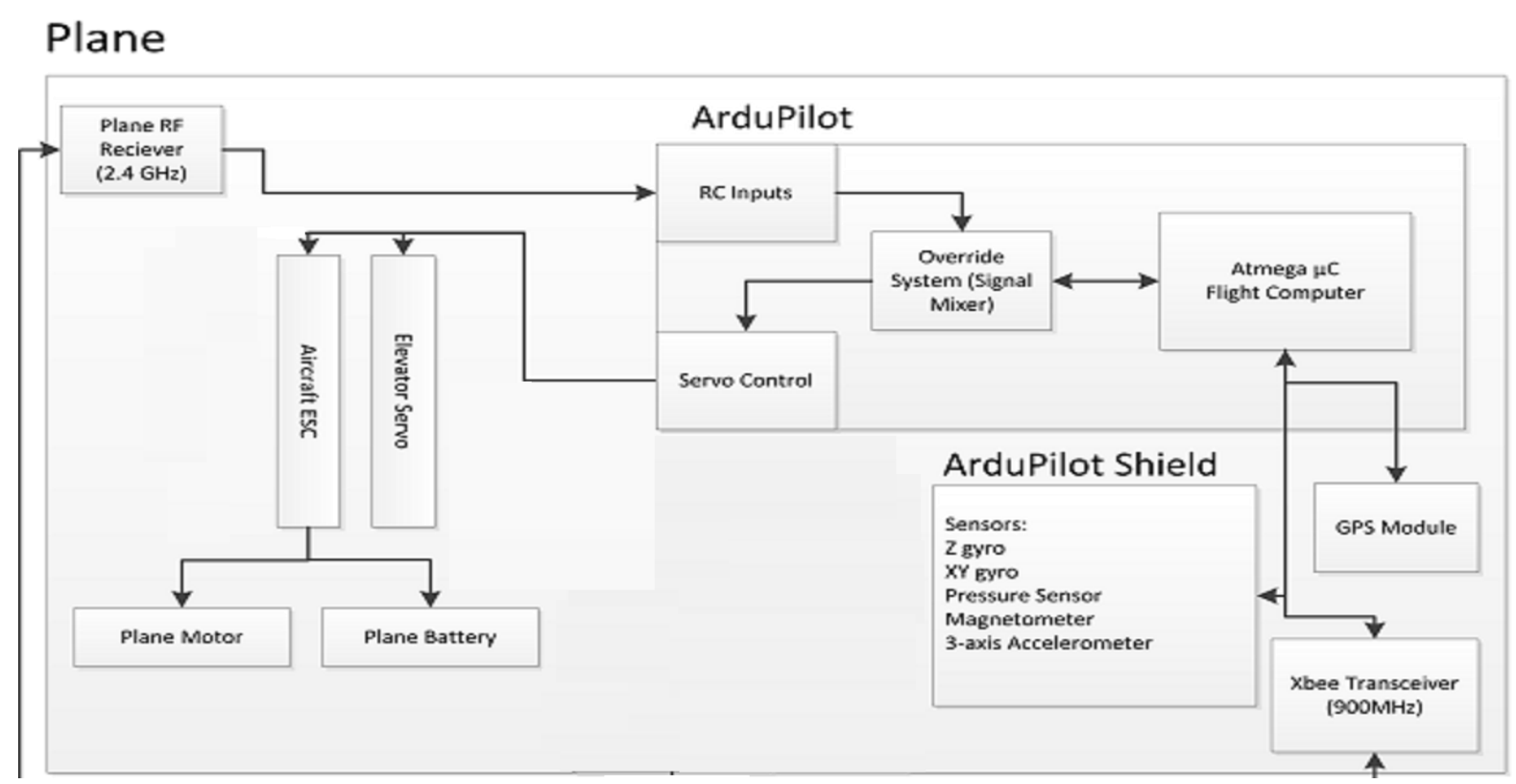

Fig. 4 Ardupilot-Mega control structure

\section{UAV Pre-Flight Check}

The following pre-flight check routine was conducted prior to each flight:

- Check all wire connections for the Autopilot,GPS, Receiver ,camera and data logger

- Leveling the airframe with the autopilot

- Check home position and altitude.

- Check Altitude accuracy from GPS and Barometric pressure sensor.

- Calibrate Radio channels (Ch. 1-2 (Elevon), Ch. 3 (Throttle), Ch. 5 (Autopilot control)).

- Switching form Manual to stabilize and Auto mode and verifying status on GUI display.

- GPS gives a 3D fix with enough No. of Satellites.

- Check the change in control surfaces between manual and auto mode.

- Check the roll, pitch and yaw by rotating the UAV and verify the ailerons and elevator move in the correct direction to compensate for this movement. 


\section{UAV Post-Flight Data Evaluations}

\subsection{UAV Data Fields}

The recorded data are composed of many attributes. The following table details the most important ones.

Table 1 Ardupilot-Mega Mavlink data attributes

\begin{tabular}{|c|c|}
\hline UAV Data Types & Attributes \\
\hline Attitude & roll, pitch, yaw, roll speed, pitch speed, yaw speed \\
\hline Global position & $\begin{array}{l}\text { latitude, longitude, alt, relative altitude, velocity in } \mathrm{x} \text { dir, velocity in } \\
\mathrm{y} \text { dir, velocity in } \mathrm{z} \text { dir, heading }\end{array}$ \\
\hline Rc channels & chan1_raw, chan2_raw, chan3_raw,, chan5_raw \\
\hline Scaled pressure & Pressure abs , pressure difference, Temperature \\
\hline Raw IMU & xacc, yacc, zacc, xgyro,ygyro, zgyro, xmag, ymag, zmag \\
\hline VFR HUD & Airspeed , Groundspeed , climb , throttle \\
\hline Servo Output & servo1_raw, servo2_raw, servo3_raw \\
\hline PID Gains & $\begin{array}{l}\text { ARSPD_RATIO,YW2SRV_P,ELEVON_MIXING,THR_FS_VALUE, } \\
\text { RC1_TRIM,RC8_ANGLE_MAX,COMPASS_DEC,ALT_MIX,THR_ } \\
\text { MAX,RC2_REV,RC7_REV,FLTMODE3,RC7_MIN,LOG_BITMASK } \\
\text {,SR3_RC_CHAN,LIM_PITCH_MIN,ARSP2PTCH_D,KFF_PTCH2T } \\
\text { HR,SR0_EXTRA2,HDNG2RLL_IMAX,... }\end{array}$ \\
\hline
\end{tabular}

\subsection{UAV Route Tracking Evaluations}

This first step in the data exploration is very important since it validates the dataset's integrity by reviewing the UAV recorded trail is conform to the actual UAV positions, the location of each waypoints is also visually checked as shown in Fig. 5 which shows a top view (latitude and longitude) of the recorded positions [4]. The straight lines indicates the desired route for the UAV which can track in an calm weather (Wind $<10 \mathrm{~km} / \mathrm{h}$ ). We have test two airframes with the same autopilot and it is clear in Fig. 5 (a) how much the trail follow the planned route while on the other hand in Fig. 5 (b) shows too much deviation from that planned route lines specially at the loiter waypoints and turns which indicates that a PID gains refinement must be applied to the airframe of Fig. 5 (b).

\subsection{UAV Position Evaluation}

We use the MediaTek patch-on-top GPS module, which is a small $(30 \mathrm{~mm} * 16 \mathrm{~mm} * 6 \mathrm{~mm})$ and light weight ( 7 grams) GPS .This GPS is compatible with ArduPilot board with data rate 10 Hz. The GPS guarantees a 2D accuracy of approximate 3m (GPS data sheet) in single GPS mode. It is possible to achieve higher accuracy using dual GPS (DGPS), where an additional GPS antenna is placed in a known location on the ground. We have test the GPS accuracy during experimental flights to see how much the GPS accuracy could influence the aircraft planned route as shown in Fig. 6, on a Google earth satellite map the yellow line shows the planned route and the black one shows the aircraft actual route, by measuring the offset between them it shows that the GPS accuracy between $3 \mathrm{~m}$ to $9 \mathrm{~m}$ which is not the same as mentioned on the GPS data sheet.

We have tested the GPS satellite fix during many experimental flights and as shown in Fig. 7, the number of satellites received by the GPS during the flight varies from turns to straight level routes. The satellites number decreases during turns while in straight level route the satellites number increased .Turn during flights decrease the sats number up to 3 . 


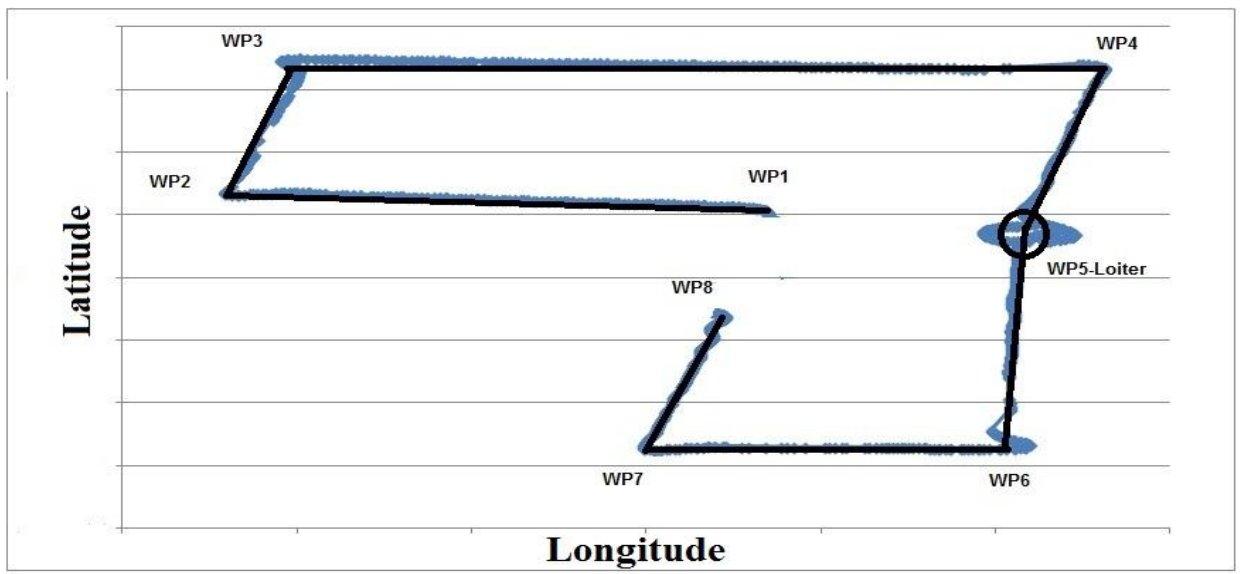

(a)

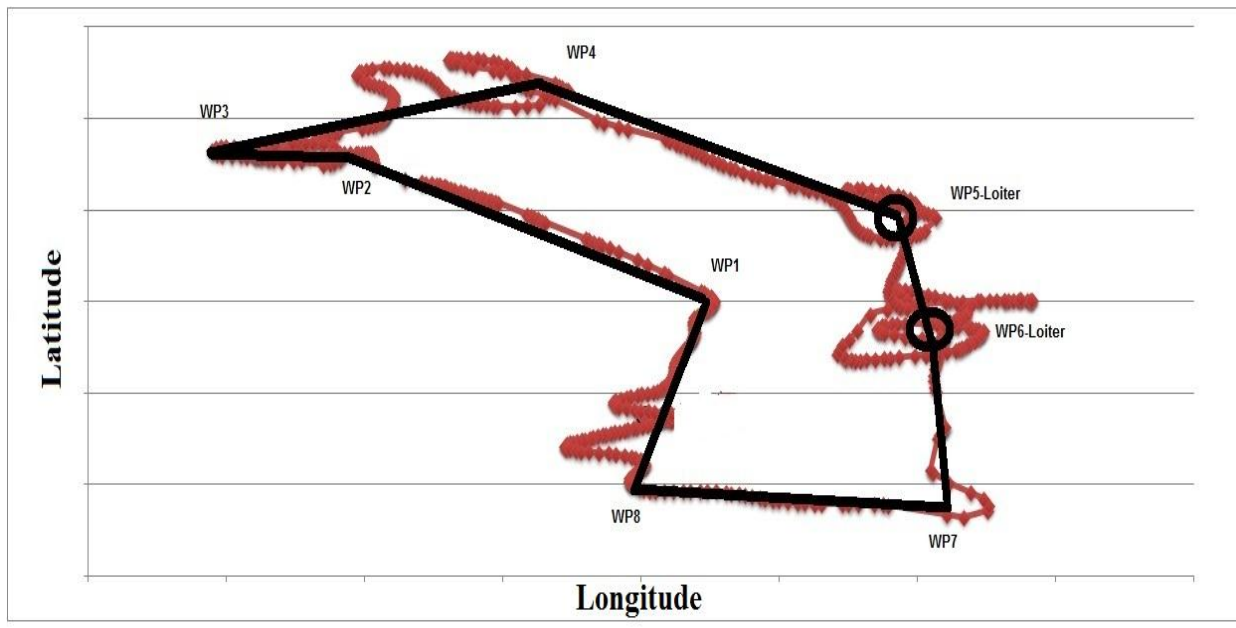

(b)

Fig. 5 Waypoint tracking error evaluation

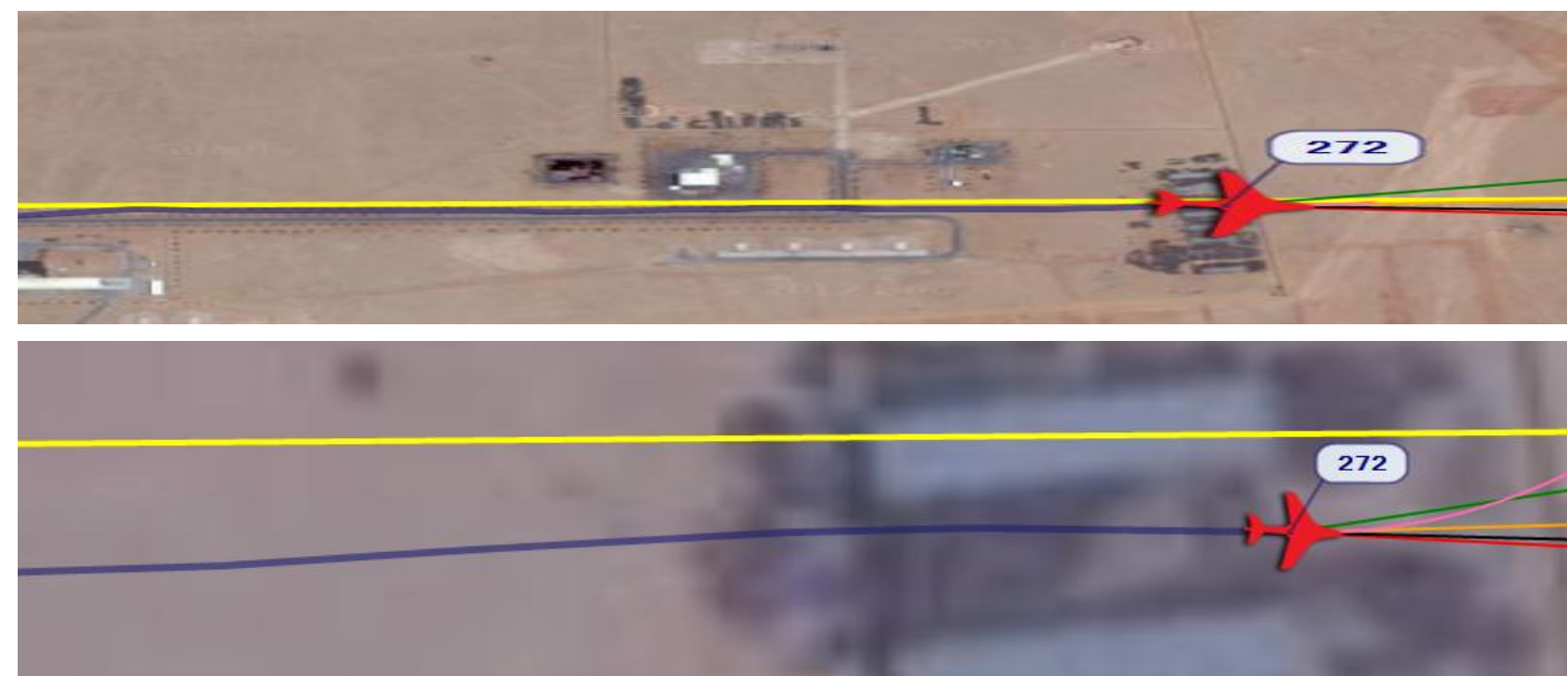

Fig. 6 Planned route(Yellow) \& mission route (Black) 


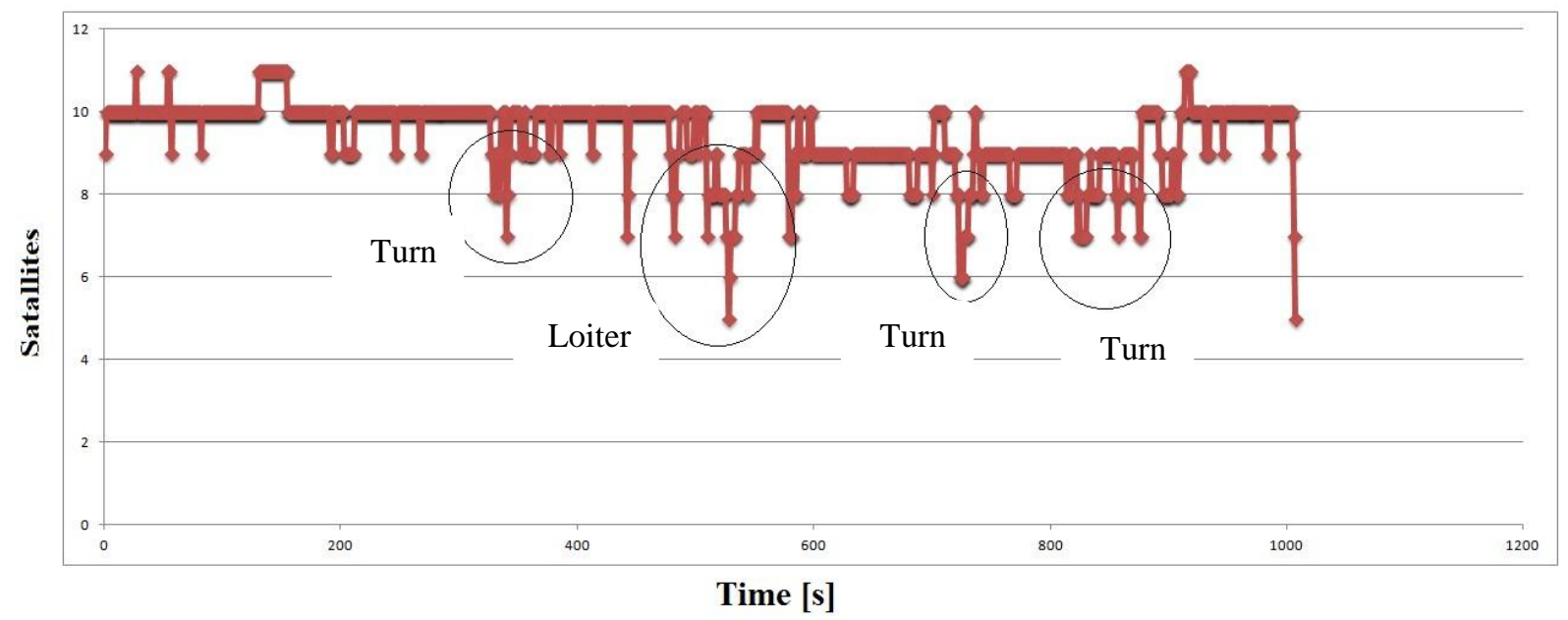

Fig. 7 Satellites number during flight route

\subsection{UAV Altitude Evaluation}

A problem with GPS 3D positioning in the altitude measurements is worse than the accuracy in the horizontal plane. Altitude is a very import parameter in flight planning procedure to evaluate the altitude data we must test all the sensors that can calculate that type of data [5]. Figure 8 shows the Altitude profile of a flight during auto flight mode.

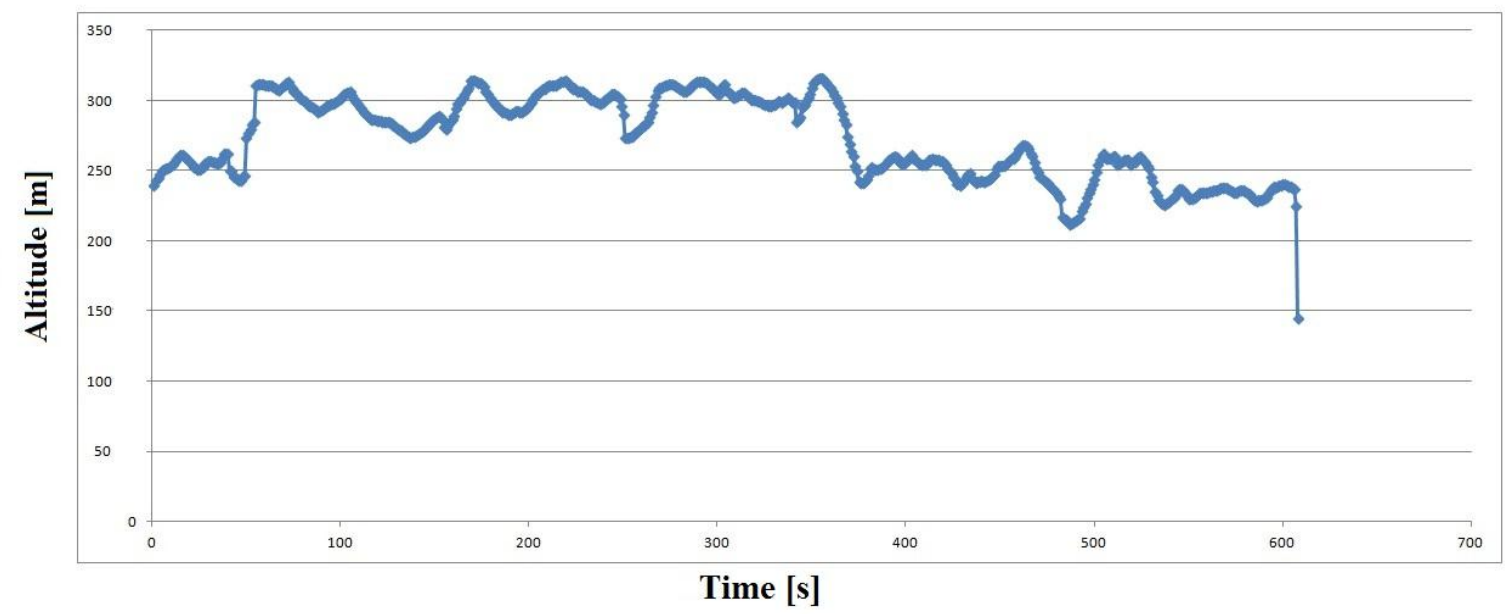

Fig. 8 Altitude evolution over time

To solve the poor accuracy of the GPS altitude measurements we use a barometric pressure sensor, During a test flight we have tested the altitude difference between the barometric pressure sensor and GPS altitude as shown in Fig. 9. The GPS altitude value oscillates rapidly and has an altitude error difference from the barometric pressure one in approximate 35 meters which shows that using GPS altitude data to the UAV could lead to unexpected behavior. 


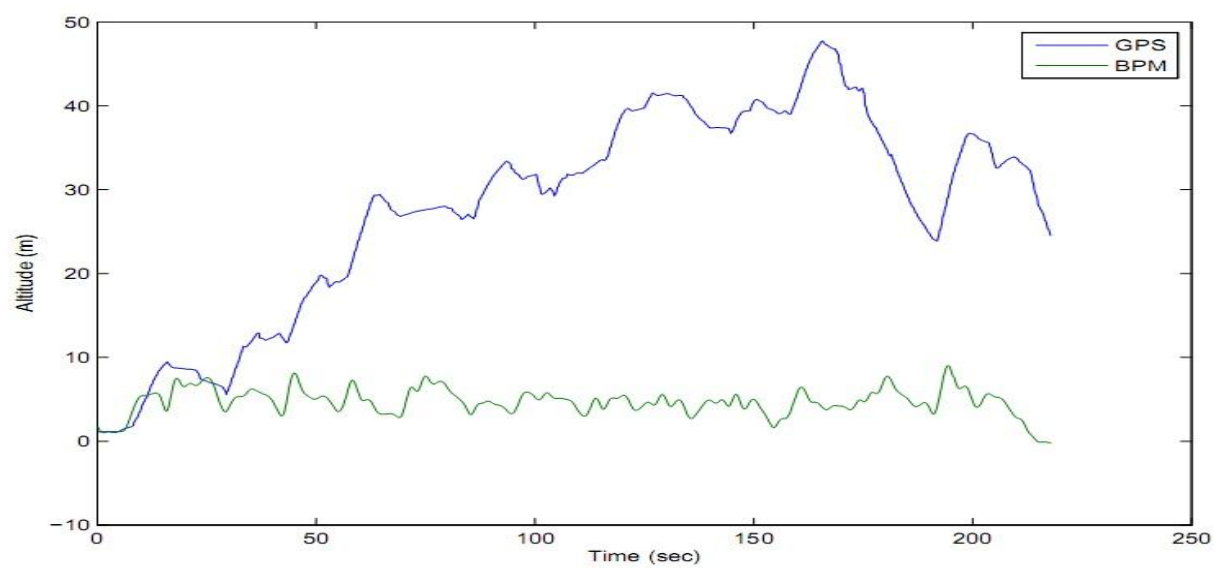

Fig. 9 Comparison of altitude accuracy between BPM GPS

The relative accuracy of the barometric pressure sensor is good but in order to get more accurate altitude during landing phase we can use a sonar sensor [6]. We compare the barometric and sonar altitude data and the sonar sensor enhance the altitude value with approximate $3 \mathrm{~m}$ as shown in Fig. 10.

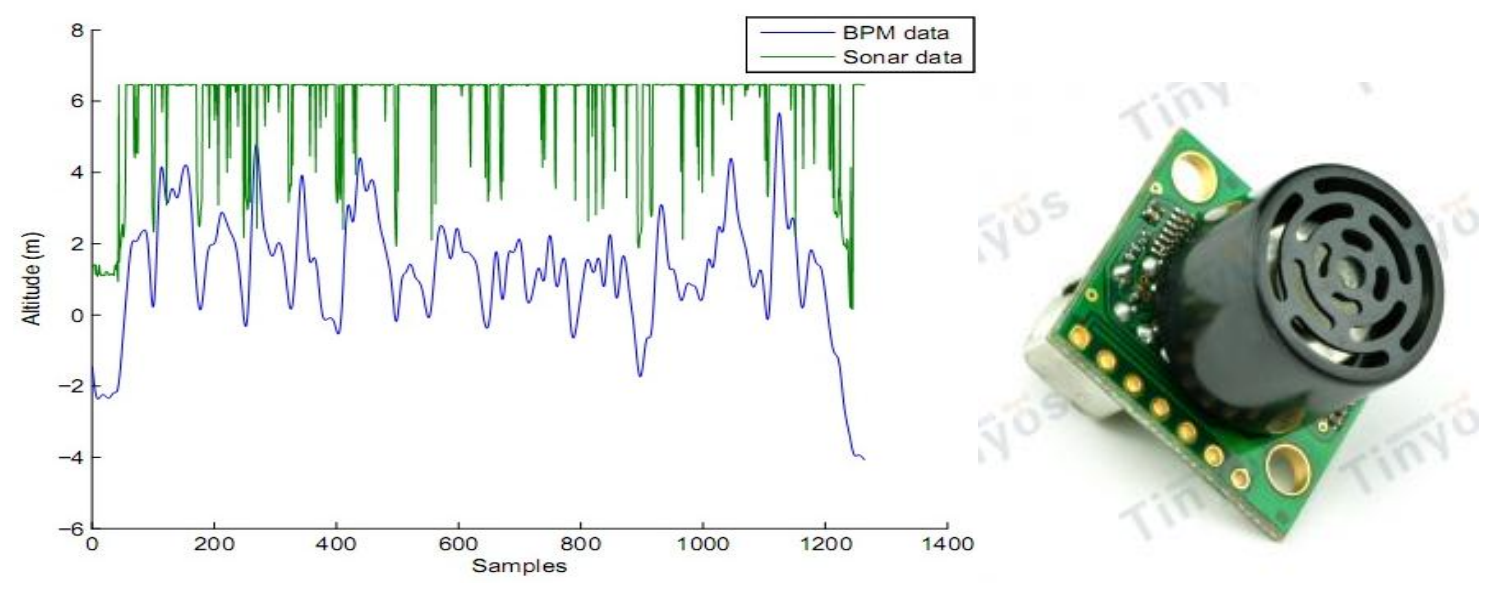

Fig.10 Comparison of Sonar (XL-Maxsonar) and BPM altitude data

\subsection{UAV Control Inputs Evaluation}

Figures 11 and 12 illustrate the control structures for stabilizing and navigation concerning pitch and banking dynamics, respectively.
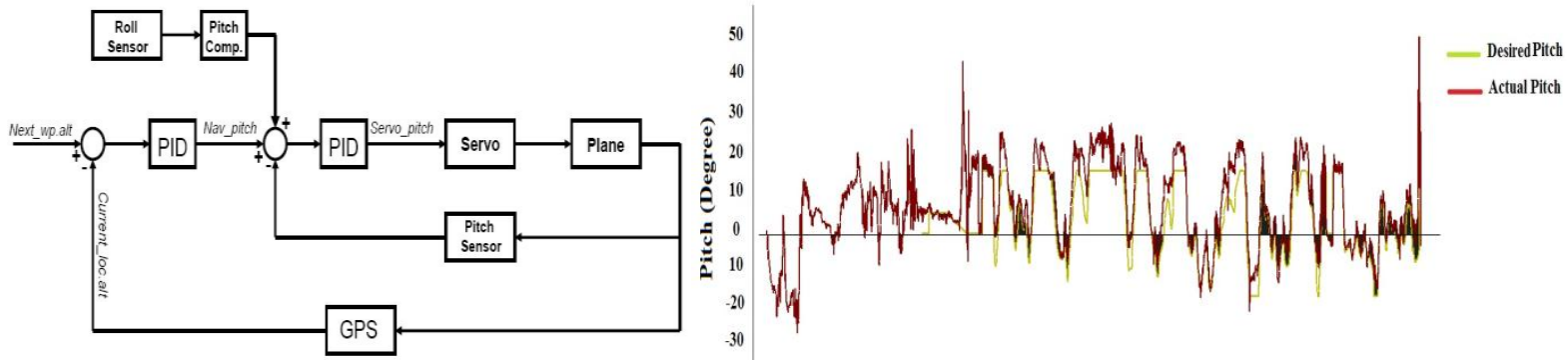

Fig. 11 Block diagram of the pitch angle control structure. 

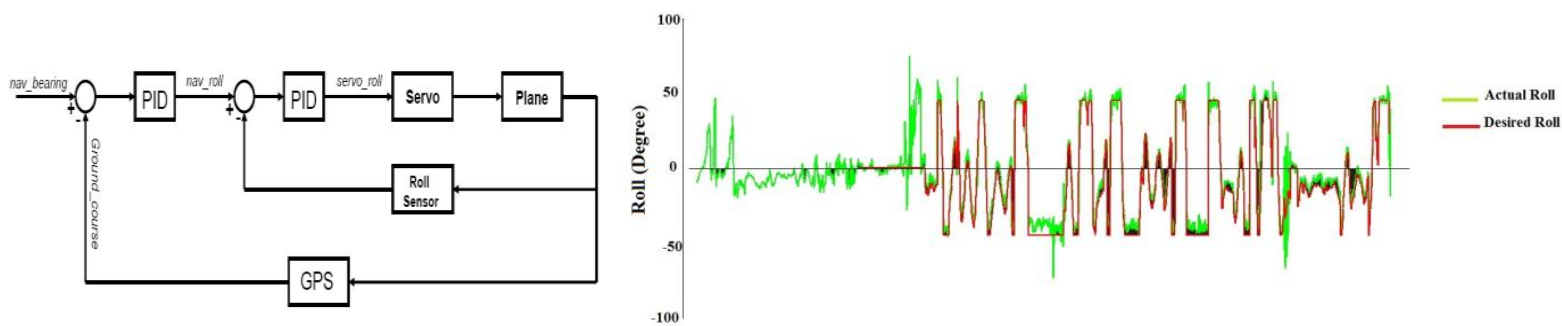

Fig. 12 Block diagram of the banking control structure used in the ArduPilot software.

The control inputs are given in the form of PWM commands that is forward directly to the servos and throttle [7]. The PWM commands need to be converted to a deflection angles, but the mapping between the control input and the deflection angle is not necessary linear ,the following equations (2) (3) describes the relation between them, Aileron Deflection Angle ( Rad)

$$
\partial_{a}=\left(9.1945 * 10^{-7}\right) u^{2}-0.0048 \mathrm{u}+5.2353
$$

where $\partial_{a}$ is the deflection angle in radians and $\mathrm{u}$ is the aiteron input

Elevator Deflection Angle (Rad)

$$
\partial_{e}=(\mathrm{u}-900 / 1200) *(1.2387+1.2051)-1.2387
$$

where $\partial_{e}$ is the deflection angle in radians and $\mathrm{u}$ is the elevator input

Integrating an autopilot boards with aircraft airframe is not an easy job, for that we have use our flight data analysis tools to check how much the airframe can be stabilized automatically by the autopilot. The following figure (Fig. 13) shows the error difference between the navigation bearing and the target bearing.

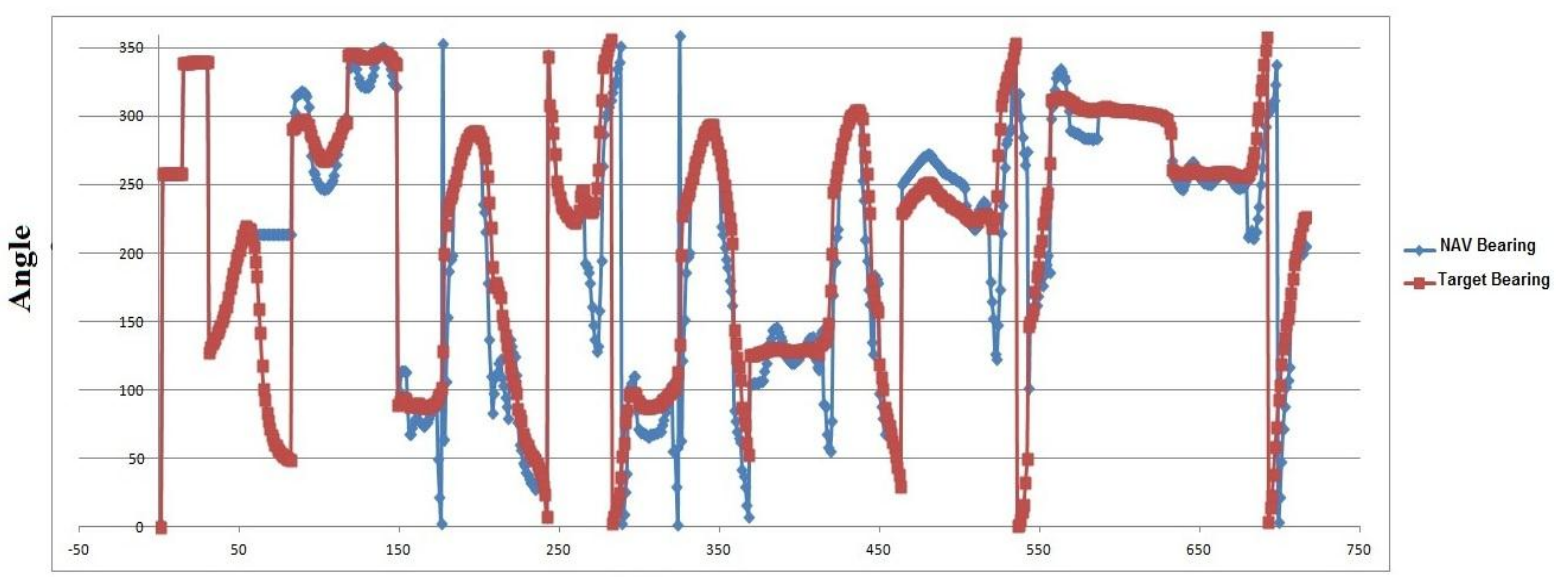

Time $[s]$

Fig. 13 Plotting of the navigation and target bearing 


\subsection{UAV Thrust Evaluation}

The throttle command are also a PWM signal and need to be converted to Newtons .The relation between the PWM and the thrust value is shown in equation (3)

$$
\mathrm{F}=\left(6.2628 * 10^{-6} * \mathrm{pwm}^{2}\right)+(0.0287 * \mathrm{pwm})-22.6335
$$

By plotting the throttle percentage during various flight modes as shown in Fig. 14, the auto pilot controls the throttle value depend on the flight plan i.e Turns, Loiter, Climb, Desent

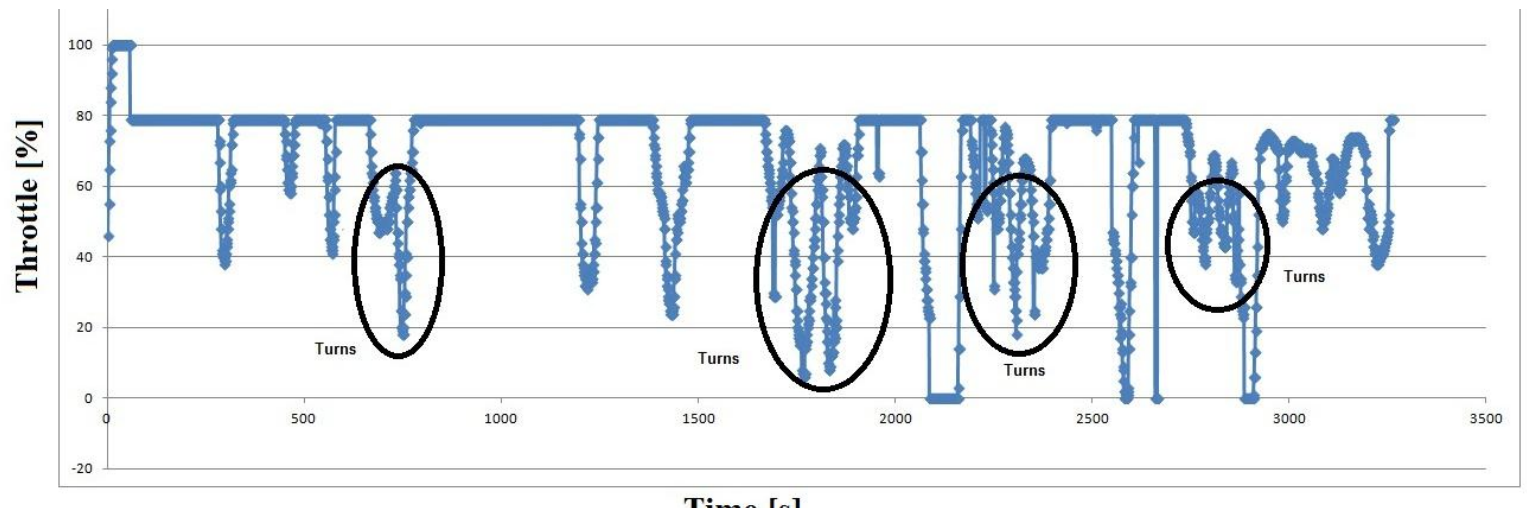

Time [s]

Fig. 14 Throttle percentage during manual and auto flight mode

\section{Conclusion and Future work}

The designed SUAV provided a good platform for the development of a UAV system. Its flight characteristics and physical dimensions meet needed criteria with reasonable constraints. Autopilot, imaging and onboard subsystems are key elements in the design. The autopilot promotes UAV usage and provides appropriate communication with the ground station. After the tuning of the control loops was completed, the overall flight performance of the autopilot and aircraft was reasonable and sufficient for the initial testing phase of this UAS. Several areas still need improvement, primarily the altitude and airspeed tracking control loops. Some of the navigation parameters could benefit from more fine-tuning. With more testing and tuning of the existing configuration, a significant improvement in performance is expected. Overall the process outlined in this paper, of integrating the Ardupilot-Mega autopilot into the selected airframe, was a success and this systems integration will allow the further development of the UAV platform to be used.

Power consumption is another issue worthy of considering, presently the power rating of the UAV will last only for several minutes without recharging, it would be great if the power rating of the UAV could be reasonably increased. The actual turning radius is affected by many factors such as wind speed, wind direction and GPS accuracy. Our recommendations for future work would be to include a more sensitive sensor for measuring altitude. For example, barometer or long range ultra-sound sensors can give more precise altitude measurements. With improved altitude sensor, the aircraft can conduct a better level turn. A better GPS sensor will also improve location tracking and autonomous navigation performance. 


\section{References}

[1] M. Y. Zakaria, Moatassem M. Abdallah, M Adnan Elshafie, "Design and Production of small Tailless Unmanned Aerial Vehicle", 15th International Conference On Applied Mechanics and Mechanical Engineering, May, 29 - 31, 2012.

[2] http://www.diydrones.com DIY Drones

[3] Justice Amahah, "The Design of an Unmanned Aerial Vehicle Based on the ArduPilot", Georgian Electronic Scientific Journal: Computer Science and Telecommunications 2009, No.5(22)

[4] David Erdos, and Steve E. Watkins, "UAV Autopilot Integration and Testing", Electrical and Computer Engineering, Missouri University of Science and Technology, Rolla, 2008.

[5] Fong Ming Yang Eugene, "UAV Flight Test System", Department of Mechanical Engineering, National University of Singapore, 2008

[6] http://www.dfrobot.com/image/data/SEN0089/MB1200-MB1300_Datasheet.pdf

[7] Eric R. Mueller,"Hardware-in-the-loop Simulation Design for Evaluation of Unmanned Aerial Vehicle Control Systems", AIAA Modeling and Simulation Technologies Conference and Exhibit, 20 - 23 August 2007, Hilton Head, South Carolina. 